\title{
Circuit
}

Musiques contemporaines

\section{Le malentendu pointilliste}

\section{The Pointillist Misunderstanding}

\section{Peter Stadlen et Nicolas Donin}

Volume 15, numéro 1, 2004

Interpréter la musique (d')aujourd'hui

URI : https://id.erudit.org/iderudit/902339ar

DOI : https://doi.org/10.7202/902339ar

Aller au sommaire du numéro

Éditeur(s)

Les Presses de l'Université de Montréal

ISSN

1183-1693 (imprimé)

1488-9692 (numérique)

Découvrir la revue

Citer cet article

Stadlen, P. \& Donin, N. (2004). Le malentendu pointilliste. Circuit, 15(1), 27-40. https://doi.org/10.7202/902339ar

\section{Résumé de l'article}

Ce texte est la traduction française de la conférence donnée par le pianiste et musicologue Peter Stadlen lors du Congrès Webern qui eut lieu à Vienne en mars 1972. L'auteur montrait tout d'abord que dans plusieurs oeuvres de la fin de la production de Webern, la prédilection du compositeur pour le palindrome musical joua un rôle déterminant dans l'organisation des séries de hauteurs et de durées. Mais à ceux qui ne voyaient en Webern qu'un mathématicien froid et abstrait, il faisait ensuite découvrir, à la lumière des informations recueillies auprès du compositeur alors qu'il travaillait avec lui les Variations pour piano op. 27, en vue de la création qui eut lieu à Vienne le 26 octobre 1937, un « poète lyrique » soucieux d'expressivité.
Ce document est protégé par la loi sur le droit d'auteur. L'utilisation des services d'Érudit (y compris la reproduction) est assujettie à sa politique d'utilisation que vous pouvez consulter en ligne.

https://apropos.erudit.org/fr/usagers/politique-dutilisation/ 


\section{Le malentendu pointilliste}

Peter Stadlen

Introduction de Nicolas Donin

\section{Éléments de contexte}

Peter Stadlen, "Das pointillistische Mißverständnis", Webern-Kongress Beiträge 1972/1973 (Österreichische Gesellschaft für Musik, ed.), Kassel, Bärenreiter, 1973, p. 173.

Peter Stadlen (1910-1996), musicien autrichien émigré en Angleterre, a mené une carrière de pianiste (à partir des années 1930), de critique musical (au Daily Telegraph, de 1959 à 1986) et de musicologue (notamment par des articles sur la question du tempo chez Beethoven et chez Schubert). Interprète dans tous les sens du terme, donc, il est particulièrement connu des historiens de la musique d'avant-garde pour avoir polémiqué, à la fin des années 1950, sur la façon de jouer Webern, controverse dans laquelle ses qualités de pianiste et d'herméneute se renforcèrent l'une l'autre adroitement.

Il avait donné les Variationen op. 27 en première audition à Vienne, le 26 octobre 1937, après les avoir travaillées de près avec le compositeur. De 1948 à 1951, invité chaque été comme professeur de piano aux cours de Darmstadt (il y donna l'op. 27 en création allemande en juillet 1948), il fera déjà figure d'ancien de la Seconde École de Vienne - bien que plus jeune que Kolisch, Steuermann ou Krenek - par rapport à la génération dite «de 1925", qui ne connut les trois Viennois que par leurs œuvres. Son jeu dut bien vite paraître ampoulé et confus en comparaison de la nouvelle norme d'interprétation qui se mettait en place à la faveur des Ferienkurse - alignant, sans crainte de l'ana- 
1. Qui donneront lieu à une édition critique de la part de Stadlen, à quarante ans de distance de l'édition originale chez Universal : WEBERN, A. (1979), Variationen für Klavier op. 27. Weberns interpretationsvorstellungen erstmals erlaütert von Peter Stadlen an Hand des Faksimiles seines Arbeitsexemplares mit Anweisungen Weberns für die Uraufführung, Vienne, Universal Edition, $29 \mathrm{p}$.

2. En particulier dans les pays francophones, puisque, à notre connaissance, aucun de ses textes n'avait été traduit jusqu'à aujourd'hui. chronisme, le dodécaphonisme des pères sur le sérialisme radicalisé fraîchement élaboré par Nono, Boulez, Pousseur ou Stockhausen. Assumant la posture réactionnaire qui lui était ainsi plus ou moins inéluctablement assignée, Stadlen défendit avec acharnement pendant le reste de sa vie la thèse d'un Webern "poète lyrique», s'appuyant sur les traces de son travail de répétition avec le compositeur pour susciter un conflit d'autorité entre la parole de Webern (dont il était dépositaire), et la lecture "pointilliste» de son œuvre (qui justifiait l'admiration sans bornes que les jeunes compositeurs lui vouaient).

Le texte qu'on va lire, postérieur à la polémique en question, est l'un des principaux textes de Stadlen sur ce sujet. Il pose, sans en avoir toujours l'air, des questions essentielles : celle du bon usage du témoignage; celle de l'engagement de l'interprète dans la diffusion de l'œuvre; celle de la performativité analytique de l'interprétation; celle, enfin (mais le décompte n'est pas clos...), de l'équilibre instable entre une mémoire inscrite dans des traces (en l'occurrence des annotations sur partition ${ }^{1}$, mises en discussion par leur confrontation avec la musicologie wébernienne des années 1960) et une mémoire sans hypostase (Stadlen ne se contente d'ailleurs pas de solliciter sa mémoire, il va jusqu'à faire parler le mort, disant en substance : si Webern avait pressenti quel tour prendrait sa postérité - et, avec elle, le cours de l'histoire de la musique moderne -, il aurait aussi précisé ceci à tel endroit, noté cela, etc.).

Pourquoi exhumer ce texte aujourd'hui? Tout d'abord pour fournir à ce numéro sur l'interprétation un cas exemplaire dans lequel on puisse scruter, comme grossis à la loupe, les principaux problèmes de l'interprétation (au double sens d'exécution et d'analyse musicale) de la musique écrite lorsqu'elle se réfere à une méthode de composition suffisamment formalisée - problèmes brusquement mis en évidence en l'occurrence par le changement d'époque dû à la guerre et à la reconstruction, impliquant accélération de l'innovation musicale et crise de la transmission.

Il s'agissait aussi de donner une profondeur de champ historique aux débats contemporains dont le présent numéro se fait l'écho, en remontant au nœud moderniste des années 1950 - racine de la «musique contemporaine» telle qu'elle s'est développée jusqu'aux années 1980, et sur fond de quoi se jouent bien des discussions et combats actuels. En effet, l'oubli dans lequel est bien vite tombée la polémique lancée par Stadlen ${ }^{2}$ a au moins un point commun avec la façon dont les divers microcosmes nationaux de la composition savante réinventent régulièrement les disputes d'il y a un demi-siècle (autour du divorce entre intellect et perception, ou sur l'usage musical de la technologie) : ces deux phénomènes - l'oubli et le ressassement passionnel - relèvent d'une même incapacité, la nôtre, à faire de ce passé un véritable objet d'histoire. 
Enfin, et pour faire suite à ce dernier point, la publication de ce texte prélude à un prochain numéro de Circuit entièrement consacré au rôle mythique joué par les cours d'été de Darmstadt dans l'imaginaire de la musique d'avantgarde depuis cinquante ans. Ce numéro ${ }^{3}$, qui mêlera témoignages actuels et approches historiques, proposera notamment un texte sur l'enjeu des lectures de Webern dans le Darmstadt des années 1950.

Nicolas Donin

LE MOT « POINTILLISTE » ne veut pas dire la même chose en musique et en peinture. Chez Seurat, une surface est peinte au moyen de points de couleurs séparés qui se fondent ensuite en une impression continue dans l'œil de l'observateur. Ce qui, en musique, se rapproche le plus de cette technique serait le système actuel de composition qui procède par textures et surfaces, ne recherchant que l'impression globale et dissimulant par contre consciemment le sort individuel des sons.

Le terme «pointilliste» - ou "ponctuel» (punktuell), pour reprendre le mot employé par Herbert Eimert en $1955^{4}$ - désigne en musique une conception selon laquelle le son individuel ne fonctionne plus comme composante d'une idée musicale. Le son n'est plus défini en fonction des rapports de tension qu'il peut avoir avec d'autres sons; il est plutôt, dans la musique purement sérielle, le point de rencontre innocent, imprévisible, de séries de paramètres fixées avant la composition, de sorte que sa hauteur n'a de sens que par rapport à d'autres hauteurs, sa durée par rapport à d'autres durées, son intensité et son timbre par rapport à d'autres intensités et timbres, et cela dans le cadre de la série.

Jusqu'à quel point peut-on démontrer une telle façon de penser chez Webern? Dans quelle mesure - pour limiter tout d'abord la question aux deux paramètres centraux - a-t-il traité séparément hauteurs et durées, c'està-dire, sans se soucier du résultat final commun? On peut déjà voir une telle tendance dans la prédilection de Webern pour le palindrome. Car même si, de façon tout à fait générale dans un palindrome musical, la rétrogradation de la deuxième moitié est imposée à la fois aux hauteurs et aux durées - la seule transformation d'ailleurs qui puisse être effectuée sur ces deux paramètres les changements résultant de la rétrogradation n'y sont cependant pas identiques : ils ont des implications harmoniques dans la sphère des hauteurs et métriques, dans celle des durées. Mais dans la mesure où l'utilisation de ce principe (la rétrogradation) donne des résultats différents pour les hauteurs et les durées, ceux-ci sont traités séparément et cet aspect de leur articulation réciproque est un effet secondaire non planifié.
3. Numéro codirigé par Jonathan Goldman et Nicolas Donin, programmé pour le deuxième trimestre 2005 (15-3).

4. Eimert, H. (1955), "Die notwendige Korrektur", Die Reihe 2, UE, p. 40. 
5. Webern, A. (1960), Der Weg zur neuen Musik, UE, p. 6o. (N. du T. : Ces leçons de Webern ont été traduites en français par Anne Servant, Didier Alluard et Cyril Huvé : Chemin vers la nouvelle musique, Paris, JCLattès, 1980, 169 p., épuisé.)

6. Dans une lettre à Erwin Stein, écrite entre le 8 et le 31 mai 1939; publiée dans : Döhl, Friedhelm (1972), "Zum Formbegriff Weberns", Österreichische Musikzeitschrift, cahier 3, p. 134 et suiv. Également, mais de façon indirecte, dans Reich, Willi et Ursula v. Rauchhaupt (1971), livret accompagnant le coffret de disques DGG 2720 029, p. 131 et suiv.; utilisée pour la première fois, selon l'affirmation de Döhl, dans sa dissertation (dactylographiée), Göttingen, 1967.
On peut tirer d'autres conclusions si l'on observe comment Webern cherche à concilier palindrome et dodécaphonisme - cela ne va pas de soi si l'on considère le fait qu'une série de douze sons ne peut en soi former un palindrome. On trouve la solution la plus simple à ce problème dans le Trio à cordes op. 20/1, mes. 1-3, où la rétrogradation n'entre en jeu qu'à la deuxième apparition des séries de durées et de hauteurs. De plus, le palindrome des mesures 1-7 des Variations pour piano op. 27/1 est dodécaphoniquement rétrograde en cela que deux formes d'une série de hauteurs, l'original et le rétrograde sont employées simultanément. Chacune de celles-ci passe d'une main à l'autre lors de la répétition du son 7 , de sorte que, comme nous l'avons déjà dit, chacune des deux séries de douze sons ne forme pas elle-même un palindrome; celui-ci est formé par le croisement du parcours des deux séries prises ensemble.

Dans la Symphonie op. 21/2, mes. 1-11 (clarinette), une seule série de douze sons, en elle-même rétrograde, est combinée à un palindrome de durées. Naturellement, le deuxième hexacorde rétrograde de cette série est transposé (afin que les 12 sons puissent se présenter), de sorte qu'il se forme au moins un palindrome comprenant onze intervalles, et dont l'intervalle central (formé par les hauteurs 6 et 7) est inversé. La différence entre les systèmes de traitement des durées et des hauteurs va donc ici considérablement au-delà de l'indépendance des paramètres décrite plus haut et qui s'applique à tous les palindromes musicaux. Toutefois, la répartition de 9 durées et de 3 silences sur chaque fois 6 hauteurs donne l'impression que le contour mélodique a d'abord été dessiné, puis soumis à un rythme, et qu'il n'a été revêtu qu'ensuite des valeurs d'une série de douze sons, selon le procédé dodécaphonique de Schönberg, bien qu'il s'agisse sûrement encore ici d'une série construite par rétrogradation, supposition qui se trouve corroborée par le fait que Webern parle d'une part de «thème » et d'autre part de la nature de sa série ${ }^{5}$.

Dans le Quatuor à cordes op. 28/3, mes. 1-13 du premier violon, un palindrome de deux fois douze durées est combiné à deux séries de douze sons. La deuxième de ces séries (mes. 8-13) est le rétrograde transposé de la première. Ces séries forment donc (tout comme les hexacordes de l'op. 21/2) un palindrome qui s'étend cette fois sur vingt-trois intervalles, et dont l'intervalle central (entre les sons 12 et 13) est renversé. Curieusement, dans l'analyse qu'il en fait, Webern a toutefois préféré décrire cette deuxième forme de la série comme le renversement de la première ${ }^{6}$. Certes cela est également vrai (et possible grâce aux transformations des trois groupes B-A-C-H), mais cela équivaut à une séparation plus rigoureuse dans le traitement des durées et des hauteurs. La tendance de Webern à penser ces paramètres de façon séparée devient encore plus évidente quand il dit de la partie de violoncelle qui évolue en 
canon par rapport à celle du premier violon, qu'elle représente, dans le premier membre de phrase (mes. 2-7), le rétrograde tant des rythmes que des hauteurs des mesures 1-7 du violon, et qu'il associe par contre dans le dernier membre de phrase (8-14) le rétrograde des rythmes du premier violon (8-13) à celui des hauteurs jouées par l'alto (9-14). Cela semble plutôt tiré par les cheveux quand on songe que le violoncelle suit ici le rétrograde des rythmes de l'alto d'au moins aussi près que celui du violon, et qu'ainsi une comparaison de l'amalgame des hauteurs et des durées de la partie de violoncelle avec celui de la partie d'alto aurait été également tout à fait possible. À part cette fusion superflue (dans la description de Webern) des hauteurs de l'une des parties avec les durées d'une autre, l'impression d'une séparation de plus en plus grande dans la façon de traiter les paramètres est intensifiée par la relation 1 : I des hauteurs et des durées, bien qu'ici le conglomérat des durées jouées et des silences produise constamment un effet plutôt thématique.

On trouve la même relation $1: 1$ dans le thème des Variations op. 30, où un palindrome de durées modifié est combiné cette fois encore à une seule série de douze sons dont le deuxième hexacorde est le rétrograde renversé du premier. Les intervalles de ces deux hexacordes ne peuvent donc pas former de palindrome. Cependant, le fait qu'au moins un silence et deux points d'orgue (qui représentent bien sûr aussi des durées) ne soient pas pris en compte, et que la modification de la rétrogradation des durées corresponde à la division de la série de douze sons en trois groupes de quatre sons - ceci est réalisé par le redoublement des valeurs brèves faisant partie du groupe du milieu, tandis que les valeurs longues du premier groupe sont réduites de moitié dans le troisième groupe, qui procède par mouvement rétrograde - voilà qui donne l'impression qu'il ne s'agit plus ici d'un contour mélodique préconçu doté d'un rythme. L'affirmation de Webern : «Mais le déroulement des motifs suit ce mouvement récurrent ${ }^{7}$ » semble confirmer que cette fois, son attention s'est portée en premier lieu sur la construction de sa série, dont la structure rétrograde se reflète pour la première fois dans la sphère des durées.

Walter Kolneder n'a donc pas raison quand il nie le moindre germe de pensée sérielle chez Webern ${ }^{8}$. Par contre, les durées n'ont jamais été manipulées par Webern de façon aussi rigoureuse et conséquente que les hauteurs, et l'on ne peut absolument pas prouver l'emploi autonome (qui ne participe donc pas seulement à l'articulation) des timbres et des intensités, sans parler des paramètres encore plus périphériques, par exemple, les densités. L'inexactitude de telles analyses ${ }^{9}$ a été confirmée tout récemment, bien que par la négative, de façon tout à fait inattendue dans la publication, dont nous avons déjà fait mention, par Friedhelm Döhl de l'analyse extrêmement détaillée que Webern
7. Chemin vers la nouvelle musique, op. cit., p. 159.

8. Au cours de sa conférence : Hat Webern seriell komponiert? (Webern a-t-il composé de façon sérielle?), Congrès Webern, Vienne, mars 1972.

9. Que l'on compare, par exemple, l'analyse que Karlheinz Stockhausen fait de l'op. 28/2 dans: "Struktur und Erlebniszeit", Die Reihe 2, p. 70 et suiv.; ou : Armin Klammer, "Weberns Variationen für Klavier, op. 27/3", Die Reihe 2, p. 85 et suiv. 
10. Döhl, F. (1963), Weberns op. 27 , Melos, p. 401.

11. Stadlen, P. (1958), "No Real Casualties?", The Score Magazine, London, novembre, p. 65.

12. Le 16 octobre 1937, dix jours avant la création, Webern écrivit à Humplik (Lettres à Hildegard jone et josef Humplik, UE 1959, p. 36) qu'il " travaillait déjà sérieusement avec un pianiste de l'endroit". a faite lui-même de son opus 28. Le fait que de tels concepts n'y sont absolument pas mentionnés prouve, indirectement certes, qu'ils étaient totalement étrangers à sa pensée, et Döhl, en $1963^{10}$, n'aurait probablement pas fait remarquer que la structure s'intensifie de plus en plus dans les 18 premières mesures de l'opus $27 / 1$ (ex. 3) si, à l'époque, il avait déjà connu ce document.

Le chemin est donc encore long qui mène de Webern jusqu'au locus classicus du sérialisme, Structures de Boulez, œuvre dans laquelle le nombre douze des hauteurs de Schönberg est reporté tout à fait mécaniquement, et sur la base de la simple analogie, sur des séries de durées fixées avant la composition ainsi que sur d'autres paramètres. (J'aimerais en profiter pour revenir à mon argument, qui jusqu'ici n'a pas encore été réfuté, que l'identité d'un son avec son octave est vraie seulement dans le sens d'une harmonie fonctionnelle, et que par conséquent la réduction du principe sériel aux douze sons qui se trouvent à l'intérieur de l'octave est arbitraire et illogique, et ce tout particulièrement dans l'optique panchromatique de Schönberg lui-même ${ }^{11}$.)

Comment se fait-il alors que depuis les années 1950, on ait sans cesse tenté de retracer des pratiques sérielles chez Webern, et cela même dans ses œuvres prédodécaphoniques, de sorte qu'au cours des ans, l'analyse a dû révoquer ce qu'elle avait imputé à Webern? Le désir qu'avait la génération sérielle de découvrir ses propres traits chez son ancêtre a sans doute joué ici un rôle - un ancêtre qui, il faut bien le remarquer, a été adopté surtout pour des raisons d'affinités esthétiques, car aussi bien Boulez que Stockhausen m'ont confirmé récemment que leurs débuts sériels tirent leur origine surtout des expériences faites par Messiaen avec la rythmique contrapuntique, ou encore de son unique essai sériel dans Modes de valeurs et d'intensités.

Par un heureux hasard, il m'est permis d'affirmer que Webern est jusqu'à un certain point lui-même responsable de ce malentendu pointilliste : en effet, dans plusieurs mouvements de ses dernières œuvres instrumentales, la partition offre une image très imparfaite de ce qu'il a indiqué comme étant le sens de sa musique, et cela à de très nombreuses reprises lors de nos rencontres qui se sont étalées sur plusieurs semaines, alors qu'il travaillait avec moi les Variations pour piano op. 27 en vue de la création qui eut lieu en $1937^{12}$; ce sens ne peut absolument pas être déduit de la partition comme c'est le cas dans les œuvres antérieures.

Tout d'abord, il aurait pu y avoir dans la partition de l'opus 27 des indications plus complètes incluant les signes ( ) $\omega^{-\mathrm{V}} \mid$ dont il s'est servi dans ma partition pour indiquer les groupes qui doivent être formés dans le $3^{\mathrm{e}}$ mouvement (ex. 1), ainsi que les accents (que je redonne ici de mémoire) des mesures 3,6, $7,10,11$, ce qui nous aurait évité l'incertitude exprimée depuis lors de différentes 
façons quant à savoir quels sons (s'il doit y en avoir) vont ici ensemble; il n'est qu'à penser aux conjectures de Friedhelm Döhl (ex. 1) qui, soit dit en passant, déclara formellement que la question de l'appartenance des sons $m i$ et $\mathrm{fa}$ des mesures 4 et 5 était non résolue ${ }^{13}$, ou la nouvelle division en mesures suggérée par Rives-Jones et qu'il considère comme plus réaliste (voir dans l'exemple 1 les lignes pointillées et les chiffres indiqués entre les systèmes ${ }^{14}$.)

\section{Exemple 1}

Webern, début du $3^{\mathrm{e}}$ mouvement de l'opus 27 . Les barres de mesure en pointillé et les indications de mesure qui apparaissent entre les systèmes sont des suggestions de Rives-Jones. Toutes les autres annotations supplémentaires proviennent de Webern.
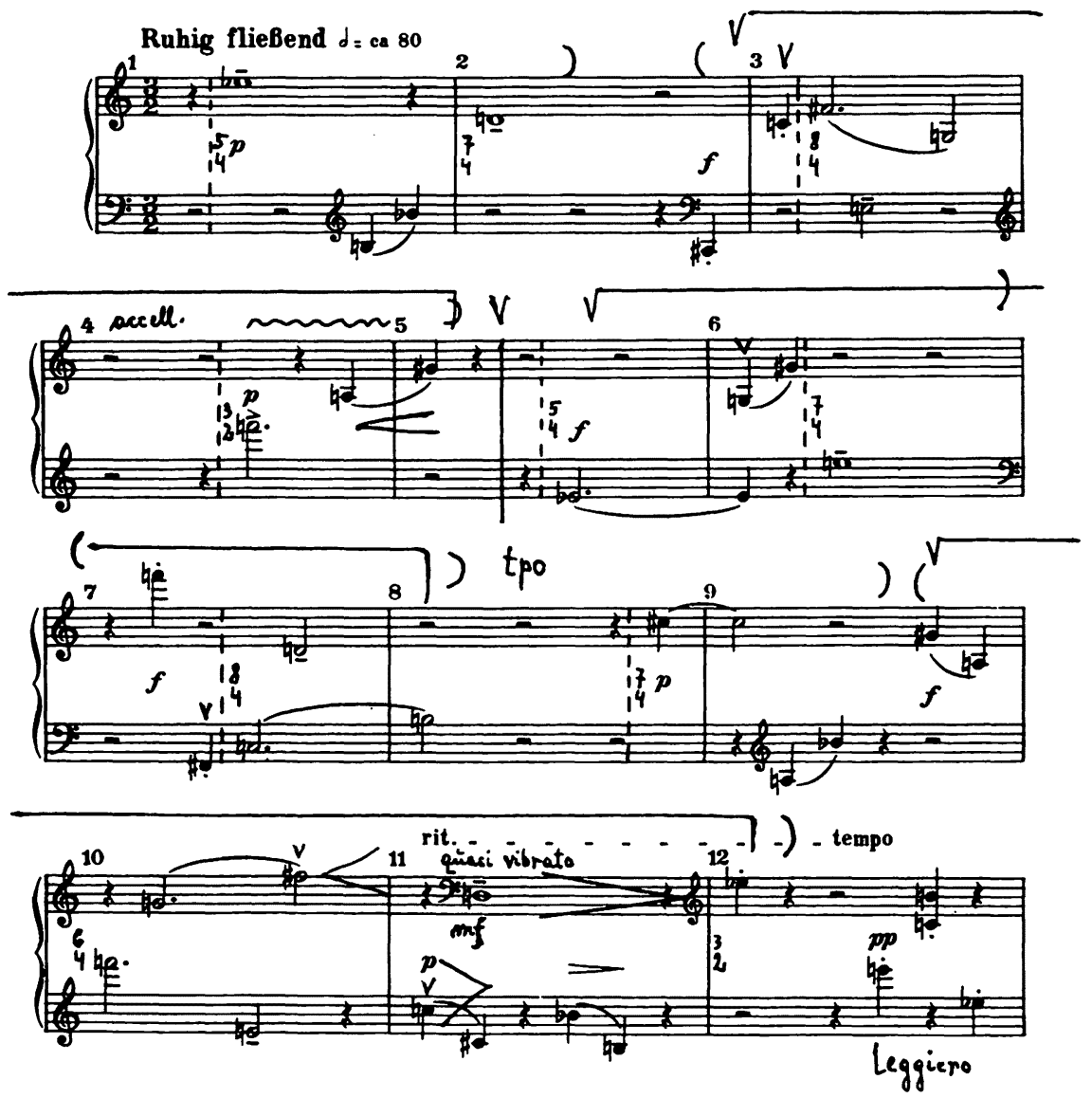

Anton Weber, Variations pour piano, op. 27

(C) 1937 by Universal Edition A.G., Wien.

www.universaledition.com
13. Döhl, op. cit., p. 403.

14. Rives-Jones, James (1968), "Some Aspects of Rhythm and Meter in Webern's op. 27 ", Perspectives of New Music, automne/hiver, p. 103 et suiv. 
Voici encore les quatre premières mesures, cette fois avec les groupements de Friedhelm Döhl.

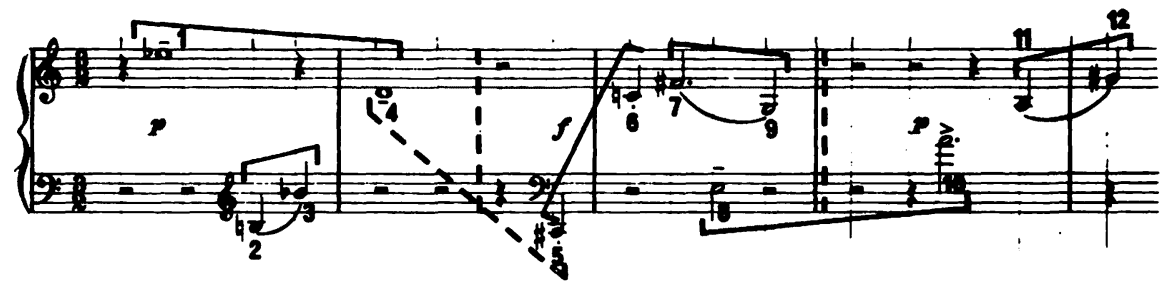

Exemple 2 : Variations, op. $27 / 2$

Sehr schnell $d=$ ca 160
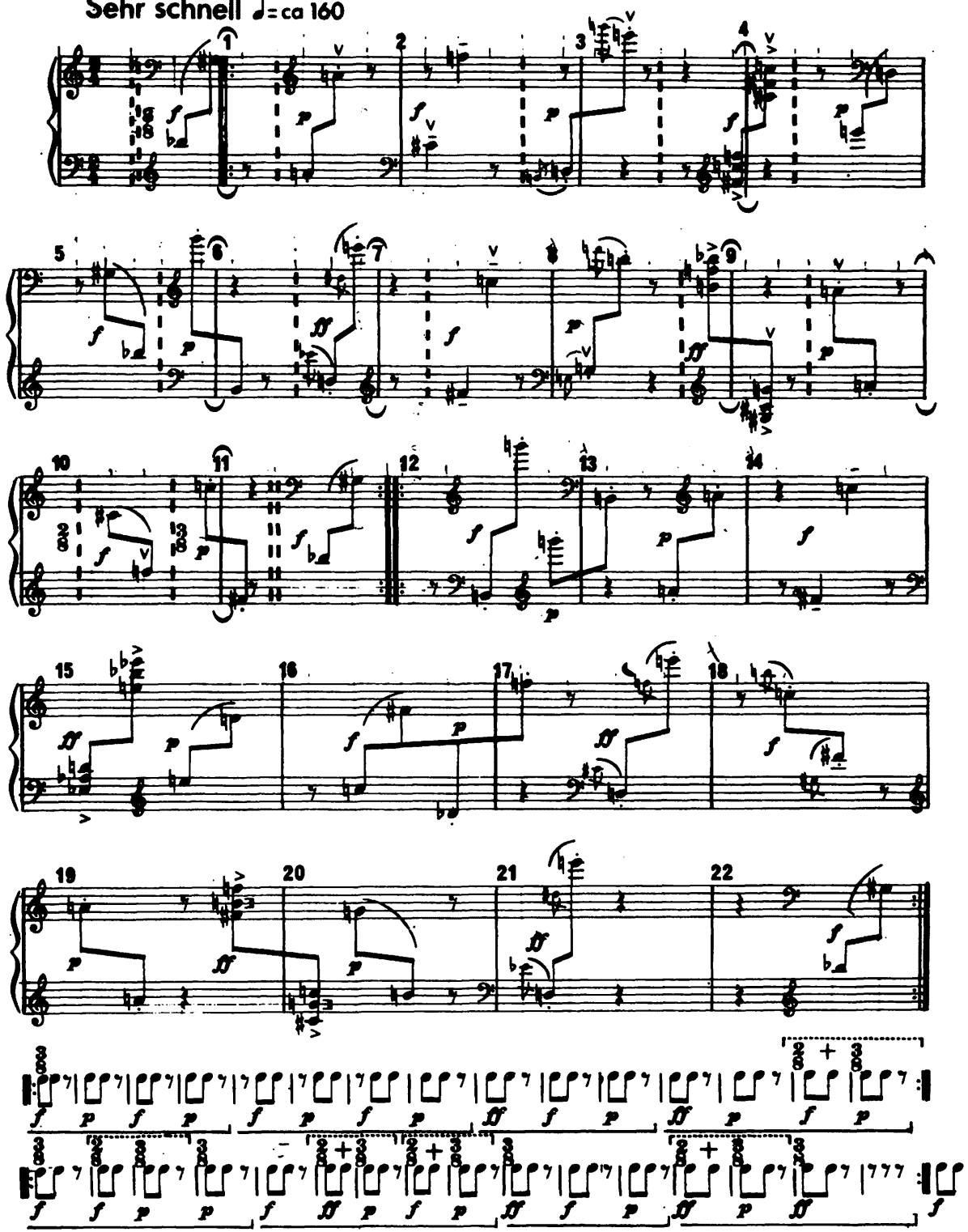
Je trouve d'ailleurs plus difficile à comprendre qu'on puisse mettre en doute les barres de mesures de Webern dans le $2^{\mathrm{e}}$ mouvement, et les suggestions de mesures à $3 / 8$ qui en résultent (voir par ex. au bas de l'exemple $2^{15}$ le schéma de mesures proposé par László Somfai et les barres de mesures en pointillé correspondantes, ainsi que les barres de mesures originales placées entre parenthèses). Elles rendent égal ce qui était inégal (voir les mes. 1 et 9,2 et 7, 2-3 et 8) et vice versa (mes. 3-4 et 8-9) au détriment des groupes de deux notes jetés pêle-mêle par Webern avec art et humour. Naturellement, ceci n'est qu'un jugement de valeur. Ce qui est plus important, c'est que même si je n'ai pas discuté de ce point avec Webern, il ne m'a jamais corrigé quand j'ai joué ce mouvement en $2 / 4$ en indiquant la mesure, tant que la composition le permet, mais en marquant tout particulièrement l'accent de la mesure 10. (Je l'ai ajouté ici de mémoire, tout comme les autres accents souhaités par Webern.) D'ailleurs, je ne me souviens pas que Webern ait voulu, dans les mesures 19-20, que les notes du milieu soient accentuées (comme l'indiquent les signes inscrits par lui dans l'exemple 2), pour marquer ainsi de toute évidence l'inversion des deux notes suivantes.

L'article de Jones est basé sur une affirmation de Edward T. Cone ${ }^{16}$ qui prétend que dans ces 12 mesures du $3^{\mathrm{e}}$ mouvement de l'opus 27 , il n'y a pas suffisamment de temps forts pour qu'on puisse reconnaître la mesure. Cette affirmation reprend une de mes remarques qui se rapportait toutefois aux mesures $25-30^{17}$ où, comme c'est si souvent le cas chez Webern, il est encore plus difficile de percevoir les syncopes du fait que deux des trois notes ou accords joués doivent l'être en ritardando.

Le rapport singulier qu'avait Webern avec le déroulement du temps en musique et qui lui faisait percevoir des variations de tempo même durant les silences, l'amenait, chaque fois que nous arrivions à la mesure 44 du troisième mouvement (une mesure vide), à prolonger l'accelerando précédent par un "eins, zwei, drei » émis avec agitation, et à indiquer ensuite, en se taisant, un point d'orgue sur la barre de mesure suivante. De ce même trait singulier provient l'accell. qu'on peut lire à la mesure 4 (ex. 1) et qui a été noté par Webern lui-même dans ma partition. De sa main est également la ligne ondulée qui suit l'accell. et qu'il avait l'habitude d'utiliser pour indiquer un léger ritenuto, ainsi que le $t[\mathrm{em}] p o$ du milieu de la mesure 8 (non précédé d'un ritardando) et le leggiero qui se trouve au-dessous de la mesure 12 . Ces indications, qu'on peut lire sur la page ici reproduite, sont les traces visibles de sa vision, une vision qui m'est restée clairement en mémoire, d'un chant à une voix pour l'essentiel (malgré les chevauchements occasionnels et les deux notes additionnelles des mesures 3 et 7 ) et qui ne passe à deux voix que dans les mesures 10 et 11 .
15. Au cours de son exposé "Rhythmic Continuity and Articulation in Webern's Instrumental works", Congrès Webern, Vienne, mars 1972. Cf. aussi Lewin, David (1963), Journal of Music Theory, printemps, p. 125 et suiv.

16. Cone, E. T. (1960), "Analysis Today", Musical Quaterly, p. 182 et suiv.

17. Stadlen, P. (1958), "Serialism Reconsidered ", The Score Magazine, février, p. 12 et suiv. 
18. Westergaard, P. (1962), "Some Problems in Rhythmic Theory", Perspectives of New Music, automne, p. 185 et suiv.

19. Westergaard, op.cit., p. 185.
Il était important pour Webern que les groupes de deux noires s'enchaînent directement avec les notes longues qui les suivent et ce, dans un caractère élégiaque entre les mes. 1 et 2 ; pathétique, dans la mes. 6 ; exalté, dans les mes. 7 et 9-10; et avec précipitation, entre les mes. 2 et 3 , où le fa dièse était perçu comme une syncope expressive.

Les notes brèves et longues ne représentent donc pas deux couches ou voix différentes et contrastantes, comme l'a présumé Peter Westergaard dans sa tentative de réponse à la question de Cone ${ }^{18}$. Quand il suppose que les deux notes isolées, c'est-à-dire celles qui ne font pas partie d'un motif de deux sons (le mi de la mes. 3 et le ré de la mes. 7), sont attirées respectivement par le fa de la mes. 4 et le do dièse de la mes. 8 , formant ainsi deux autres groupes de deux sons, il s'agit là d'une hypothèse qui n'est pas valable, comme le montrent bien les signes de phrasé de Webern déjà mentionnés. Webern a d'ailleurs communiqué ses vues en déclamant, en dirigeant et en chantant. En ce qui a trait au $m i$ bémol de la mes. 12, Webern, dans les années précédentes, aurait probablement écrit verlöschend (morendo) au-dessus de cette note, devançant ainsi la supposition de Cone qui affirme qu'on trouve ici le premier point d'ancrage structurel de ce mouvement.

Les cinq pages suivantes de ma partition contiennent d'autres signes de phrasé, quelques signes de pédale ainsi qu'un nombre important de crescendi et decrescendi, qui d'ailleurs, dans les mes. 56-57, opposent le groupe de la main gauche (crescendo) à celui de la main droite (decrescendo), montrant ainsi à quel point on peut se tromper quand, à partir de la présence de quelques indications apparues lors de l'édition, on tire des conclusions au sujet de la structure $^{19}$. Je souhaiterais pouvoir décrire par des mots - que ce soient les miens ou certains que nous a transmis Webern (tels que cantabile qui apparaît deux fois, au-dessus des mes. 13 et 22 ; hart (avec dureté) et weich (avec douceur) au-dessus des mes. 25 et 26) - et faire imaginer l'arioso brûlant, j'irais presque jusqu'à dire : opératique, que Webern a vu dans ce mouvement. À partir de la mes. 45 , les constants changements de tempo revêtent un caractère si impétueux - Höhepunkt, Vorwärts, Stark (sommet, avancer, avec force) peut-on lire au-dessus de la mes. 56 - qu'il ne reste parfois pratiquement rien du placement des valeurs de notes qui, s'il n'a pas été fait de façon systématiquement sérielle, témoigne tout de même d'un soin très méticuleux.

Le fait que Webern ait renoncé presque complètement à indiquer au pianiste que ce n'est pas un pointilliste qui s'exprime ici, mais un poète lyrique qui s'efforce véritablement d'être expressif mais à qui son propre mélodisme semble par trop bavard, a largement contribué à ce que, dans les œuvres instrumentales de la fin de sa production, là où il y a des formations et des correspondances 
indéniablement géométriques, celles-ci soient perçues comme étant une fin en soi. Même un observateur comme Adorno, qui n'était pas du tout enclin à penser en terme de musique sérielle, parle de "chosification totale ${ }^{20}$ ", tandis que Krenek voyait un contraste entre la «tension nerveuse » des Chants op. 15-19 et «l'ascèse classique, voire académique» de la série d' «œuvres instrumentales qui commence avec la Symphonie op. $2 \mathbf{1}^{21}$. " Il constatait encore récemment que Webern s'était «détourné de la peinture des sentiments humains " pour se «limiter de plus en plus, dans les op. 21 et 24, et particulièrement dans les Variations pour piano op. 27, à un monde de formes abstraites froid et autosuffisant ${ }^{22}$ ».

Cependant, dans la partie centrale du premier mouvement des Variations pour piano un style d'improvisation libre est à tout le moins suggéré par les indications imprimées relativement abondantes, même si ces indications d'exécution ne laissent pas pressentir toute l'acuité des contrastes émotionnels et les changements brusques des images et des tempi.

Ce sont les palindromes de la première et de la troisième partie de ce mouvement qui donnent le plus l'impression d'une construction qui se suffit à ellemême, et ils ont en effet mené à une exécution aussi neutre et par le fait même aussi déformante que celle de Leonard Stein, qu'on peut entendre dans l'enregistrement sur disques de l'œuvre complète réalisé par Robert Craft. Les signes de vibrato injouables des mesures 2 et 3 (ex. 3) que Webern a notés dans ma partition (de sa main également sont les lignes ondulées qui indiquent un ritardando, les traits d'allongement dans les mes. 5 et 7 et les crochets des mes. 8-10), donnent une idée du type d'expression lyrique à la fois détaché et passionné qu'il se représentait ici. Et même si dans les mes. 2, 3, 7 et 8, il a noté luimême l'«enchaînement » entre les deux mains, je sais toutefois qu'il voulait surtout que, dans les mes. 1, 2 et 6, 7, la plainte contenue et son renversement dans les notes plus aiguës soient accentués. Dans les mes. 3 à 5 , la mélodie est répartie entre les deux mains, et c'est également le cas à partir de la mes. 8; cependant, comme les notes encerclées le montrent (cette fois-ci, c'est moi qui les ai indiquées de mémoire, tout comme les crescendi, les decrescendi et le molto espressivo), cela n'est pas systématique : le do dièse de la mes. 17 fait partie de la mélodie, mais pas celui de la mes. 9, et dans la reprise, à partir de la mes. 37, la répartition est encore moins consistante et prévisible.
20. Adorno, Th. W. (1959), Klangfiguren, Suhrkamp, p. 176.

21. Melos, 1957, p. 304-305.

22. Krenek, Ernst (1966), Anton v. Webern, A Profile; Perspectives, University of Washington Press, p. 10. 


\section{Exemple 3 : Variations, op. 27/1}

Toutes les indications supplémentaires proviennent de Webern.
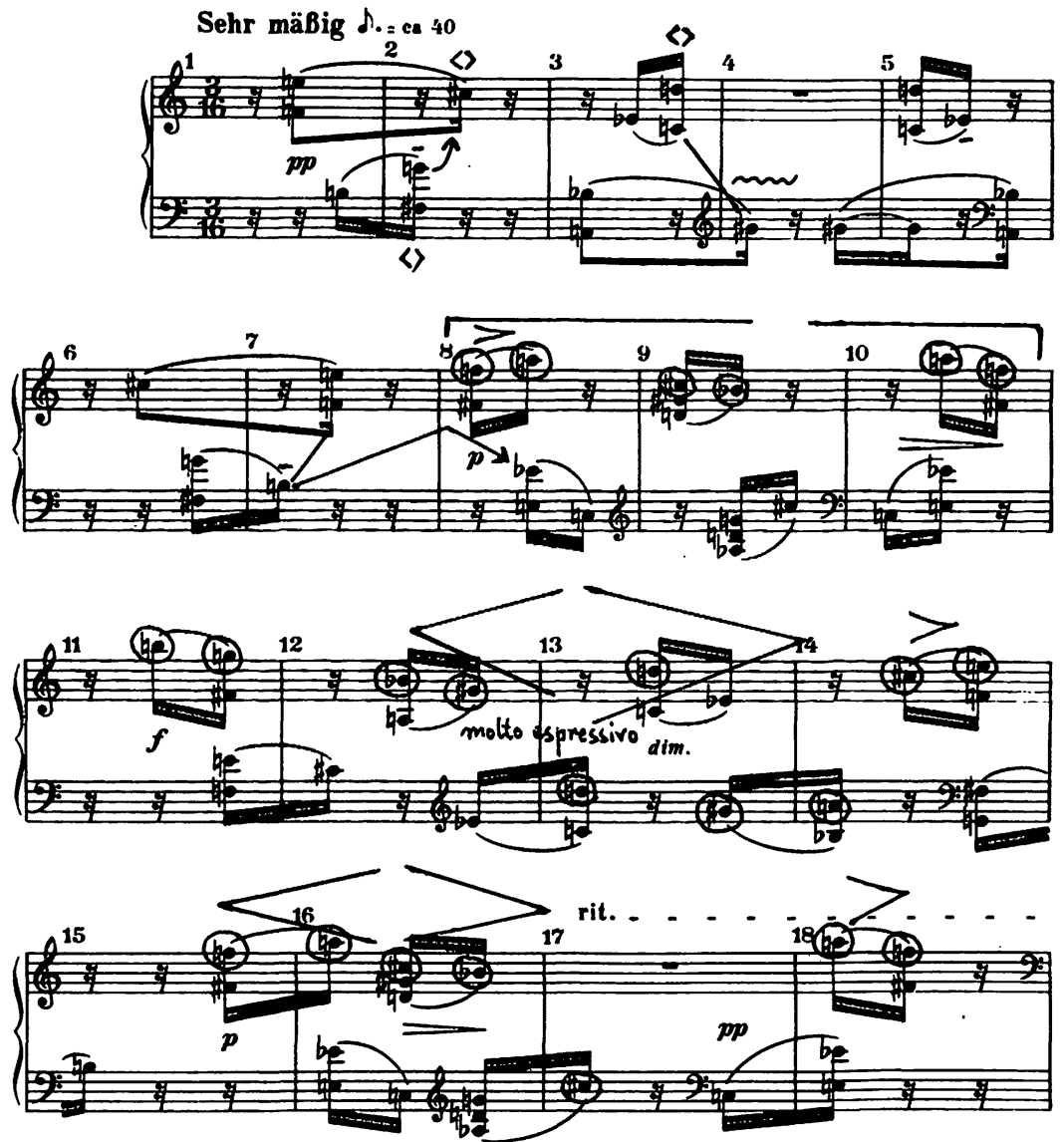

Voici l'analyse des densités que Friedhelm Döhl a faite de cette page :

Dans la partie $\mathrm{A}$, la première période correspond à la troisième, et la deuxième à la quatrième. La répartition des sons joués simultanément montre une condensation structurelle de plus en plus grande.

Mesures $1-7$ incl. $1^{\text {re }}$ période : $2121 ; 2121 ; 1212 ; 1212$.

Mesures 8-10 incl. $2^{\mathrm{e}}$ période : $231 ; 341 ; 132$.

Mesures $11-15$ première note $3^{\mathrm{e}}$ période : $141 ; 222 ; 222 ; 141$.

Mesures 15 dernière note -18 incl. $4^{\mathrm{e}}$ période : $23441 ; 132$.

Voici un cas étrange : une structure planifiée selon toutes les règles de l'art dans laquelle la plupart des sons fonctionnent seulement comme l' «accompagnement» d'une «mélodie » qui par endroits va à l'encontre de la structure. Nous savons tous à quel point il peut être irritant qu'on nous serve l'Art de la 
fugue ou l'Offrande musicale joués de façon expressive. Mais c'est justement cette dialectique entre la volonté d'expression et le vouloir constructiviste qui est caractéristique des œuvres instrumentales de la fin de la production de Webern. Si cet artiste raffiné et réservé avait annoté ses partitions avec autant de minutie que Mahler, par exemple, qu'il admirait beaucoup et dont la personnalité se reflète dans la somme d'indications d'exécution laissées dans ses partitions, les 25 dernières années de l'histoire de la musique se seraient peutêtre déroulées autrement.

\section{Discussion}

Cette discussion tente de résoudre la difficulté d'une délimitation terminologique entre «thème» et «série». Il semble qu'il ne soit pas possible d'établir une frontière et par conséquent d'en arriver à un consensus sur la question de savoir si Webern a utilisé ou non des séries de durées. La question du nombre douze n'est pas considérée comme pertinente dans ce contexte; la question de l'«utilisation systématique » de séries de durées ne peut, semble-t-il, servir de critère pour établir l'existence de ces séries. Le $\mathrm{D}^{\mathrm{r}}$ Stephan nous fait remarquer que l'expression Grundgestalt (forme de base) inventée par Schönberg peut être considérée comme un chaînon intermédiaire important entre thème et série : à ce stade déjà, l'ordre sériel et la possibilité d'une pensée symétrique seraient liés. Très souvent dans l'histoire de la musique, de nouvelles impulsions fondamentales et des œuvres importantes seraient nées d'une telle situation de transition; pour cette raison, la question de l'intention consciente n'aurait au fond pas d'importance.

(Traduction : Yves Saint-Amant) 


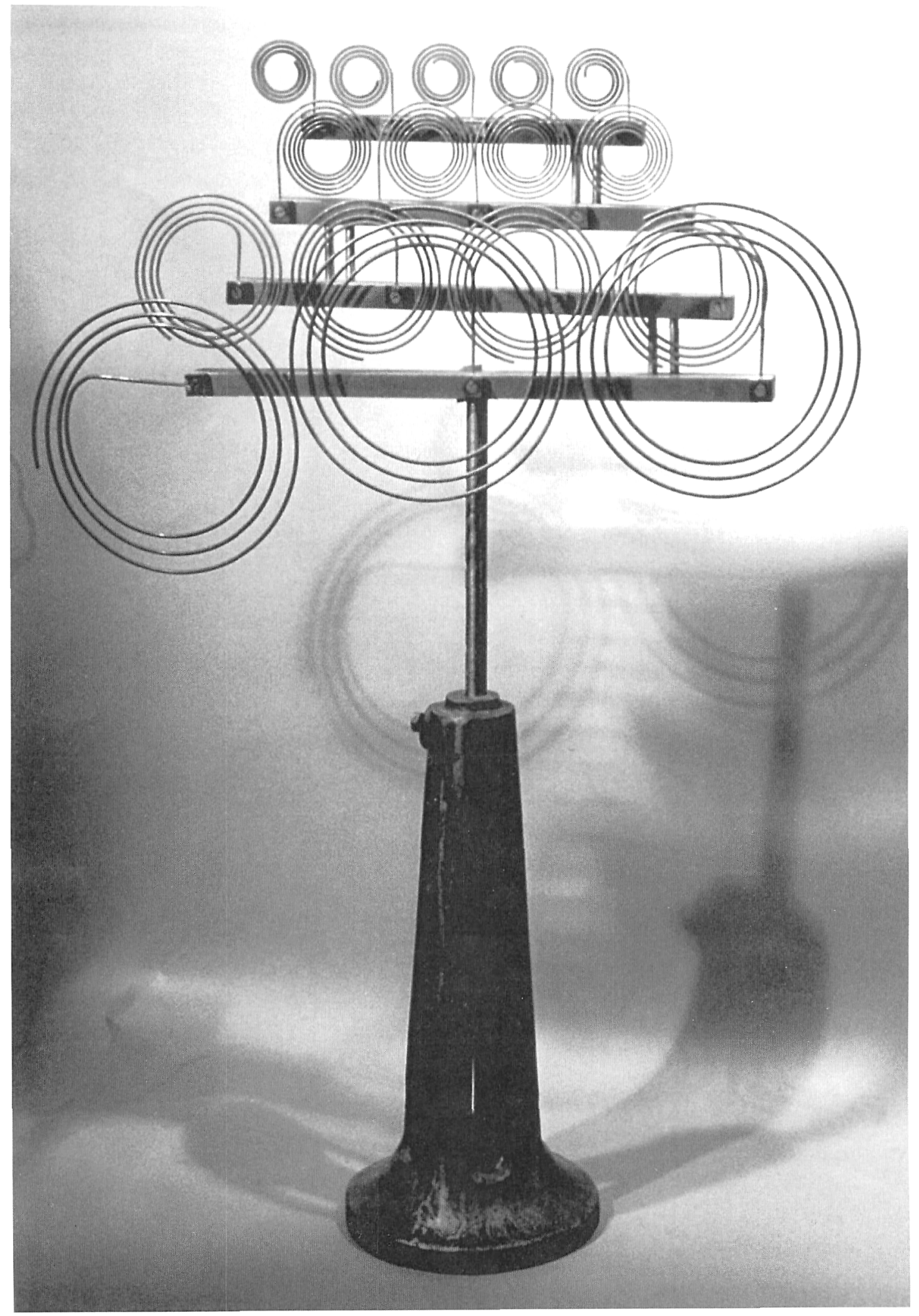

